\title{
Quasicontinuous pseudoimages in sinusoidal grating imaging using an extended light source
}

\author{
Luis Miguel Sanchez-Brea $^{\text {a,*, Jose Alonso }}{ }^{\text {a }}$, Eusebio Bernabeu ${ }^{\text {b }}$ \\ a Departamento de Optica, Universidad Complutense de Madrid, Escuela Universitaria de Optica, Avda. Arcos del Jalon s.n., \\ 28037 Madrid, Spain \\ ${ }^{\mathrm{b}}$ Departamento de Optica, Universidad Complutense de Madrid, Facultad de Ciencias Fisicas, Ciudad Universitaria s.n., \\ 28040 Madrid, Spain
}

Received 12 November 2003; received in revised form 8 March 2004; accepted 8 March 2004

\begin{abstract}
When a polychromatic light illuminates a grating, an achromatic and continuous self-imaging regime is produced, what means that Talbot planes are eliminated. In this work, we investigate the existence of this continuous regime for generalized grating imaging. We have found that amplitude gratings with sinusoidal profiles may form, under monochromatic and partially coherent illumination, pseudoimages whose contrast or modulation present a smooth dependence on the gap between the two gratings.
\end{abstract}

(c) 2004 Elsevier B.V. All rights reserved.

PACS: 42.25.Fx; 42.79.Dj

Keywords: Generalized grating imaging; Pseudoimages; Talbot effect

\section{Introduction}

There are several processes for creating images of a grating by using only gratings as imaging elements, being the most known the Talbot effect [1]. Self-images of the grating appear under monochromatic and spatially coherent light and they are located at positions $z_{N}$ given by $z_{N}=N\left(p^{2} / \lambda\right)$,

\footnotetext{
${ }^{*}$ Corresponding author. Tel.: +34-91-394-4555; fax: +34-91394-4674. Brea).

E-mail address: sanchezbrea@fis.ucm.es (L.M. Sanchez-
}

where $p$ is the period of the grating, $\lambda$ is the wavelength of illuminating light and $N$ the order of the self-image, any positive integer. The odd selfimages $(N$ odd) of a grating with aspect ratio 0.5 present contrast reversal, that is, the images are shifted by $p / 2$ with respect to the original grating. The contrast of the Talbot images decreases as coherence of the illuminating beam (both temporal or spatial) is reduced. This contrast reduction increases as the ratio $p / \lambda$ decreases, or as the order of the self-image increases. Grating self-imaging, its applications, and its effect in metrological systems using gratings have been extensively studied [2-4]. 
There are other configurations in which one grating acts as an imaging element for another grating, such as the Lau effect [5,6], where two gratings with identical periods are illuminated with a monochromatic light source with finite extension, and a pseudoimage of the first grating is formed at the focal plane of a lens located behind the second grating (that is, the image is formed at infinity). In the Lau effect, the lack of spatial coherence of the source is a key condition. The presence of many incoherent light paths leads to a simple geometrical interpretation for the image formation in the Lau configuration, but there remains a fundamental phenomenon related to the wave nature of light: the distance between the two gratings must be a Talbot distance in order that the Lau image may be formed. The period of the Lau fringes depends on the period of the gratings, the gap between them and the focal length of the lens employed.

When the periods of the gratings are not equal, a lens is no longer necessary and pseudoimages form at many different locations after the second grating. Period and transmittance profile are not equal to that of the first or second grating, so the periodic patterns formed after the second grating are named pseudoimages. The whole phenomenon is called generalized grating imaging. The effect has been investigated by a number of authors. Patorski described pseudoimages as the incoherent superposition of self-images of the second grating created by each of the slits of the first grating [7]. Swanson and Leith [8] used the source model usually employed in the analysis of the Lau effect (totally incoherent source of infinite size infinitely far away). They obtained an expression for the location of the pseudoimages, but their analysis implies that depth of field of the images is zero. Tu and Zhan, as well as Olszak and Wronkowsky used phase space distribution functions to cope with partial coherence of the source $[9,10]$. In more recent works by Crespo et al. [11,12], similar results to those of Tu and Olszak are obtained by means of a finite source model at finite distance from the first grating. These authors analyze the location, depth of focus and modulation of the pseudoimages, also giving approximate analytical expressions for these parameters, as well as an indexation scheme of the obtained pseudoimages.

Pseudoimaging has many similarities with the Lau effect, although is a more general phenomenon. One of the similarities is that the contrast of any pseudoimage strongly depends on the gap between the gratings. A condition similar (but not equal) to that of the Talbot distance must be met in order that the pseudoimages have significant contrast. The variation of this gap corresponding to two consecutive maxima in the contrast of a pseudoimage is in the order of $p^{2} / \lambda$. When the grating periods are not too much larger than the wavelength (let us say $p / \lambda \lesssim 10$ ), then $p^{2} / \lambda$ is just a few tens of microns, and tiny perturbations of the grating gap (in the order of $p^{2} / 2 \lambda$ ) may change the contrast of the pseudoimage from a maximum to near zero. Recently, Guerineau et al. have shown that a continuum of self-images of a grating can be obtained by using a polychromatic light source [13-15]. This illumination provides a continuous and achromatic self-image regime.

In this work, we investigate the existence of this continuous regime for generalized grating imaging. We have found that amplitude gratings with sinusoidal profiles may form, under monochromatic and partially coherent illumination, pseudoimages whose contrast or modulation present a smooth dependence on the gap between the two gratings. This property may lead to spectrometers or metrological devices which make use of the pseudoimaging phenomenon with extended robustness and greater tolerances.

\section{Analytical expression for sinusoidal grating imaging}

Let us consider a double grating system as depicted in Fig. 1. The gratings are named $G_{1}$ and $G_{2}$, with periods $p_{1}$ and $p_{2}$, respectively. The lateral size of the source is $S$ and we will assume it is formed by a uniform distribution of independent punctual sources of wavelength $\lambda$. We will call $z_{0}$, $z_{1}$ and $z_{2}$ to the distances between the source and the first grating, from the first to the second grating, and from the second grating to the observa- 


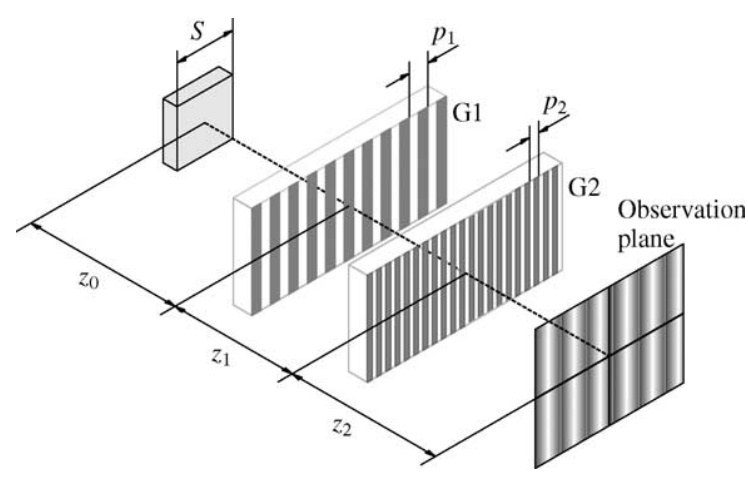

Fig. 1. Setup for generalized grating imaging, showing the parameters involved.

tion plane, respectively. According to Crespo et al. [11], the intensity distribution at the observation plane $P$ is

$$
\begin{aligned}
I(x)= & \sum_{n} \sum_{n^{\prime}} \sum_{m} \sum_{m^{\prime}} a_{n^{\prime}}^{*} b_{m} b_{m^{\prime}}^{*} \exp \left\{\mathrm { i } \left[\alpha\left(n-n^{\prime}\right)\right.\right. \\
& \left.\left.+\beta\left(m-m^{\prime}\right)\right]\right\} \times \exp \left\{\mathrm { i } \left[\gamma\left(n^{2}-n^{\prime 2}\right)\right.\right. \\
& \left.\left.+\delta\left(m^{2}-m^{\prime 2}\right)\right]\right\} \times \exp \left[\mathrm{i} \xi\left(n m-n^{\prime} m^{\prime}\right)\right] \\
& \times \operatorname{sinc}\left[\eta\left(n-n^{\prime}\right)+\tau\left(m-m^{\prime}\right)\right]
\end{aligned}
$$

where $a_{n}$ and $b_{m}$ are the Fourier coefficients of both gratings $G_{1}(x)=\sum_{n} a_{n} \exp \left(\mathrm{in} q_{1} x\right), G_{2}(x)=$ $\sum_{m} b_{m} \exp \left(\mathrm{i} m q_{2} x\right), q_{1}$ and $q_{2}$ being their respective spatial frequencies $q_{i}=2 \pi / p_{i}, i=1,2$. The other parameters in Eq. (1) are given by

$\alpha=x q_{1} z_{0} / z_{T}, \quad \beta=x q_{1} R\left(z_{0}+z_{1}\right) / z_{T}$

$\gamma=\pi z_{0}\left(z_{1}+z_{2}\right) /\left(2 z_{T} z_{\lambda}\right)$,

$\delta=\pi R^{2} z_{2}\left(z_{0}+z_{1}\right) /\left(2 z_{T} z_{\lambda}\right)$,

$\xi=\pi R z_{0} z_{2} /\left(2 z_{T} z_{\lambda}\right), \quad \eta=S q_{1}\left(z_{1}+z_{2}\right) /\left(2 z_{T}\right)$,

$\tau=S q_{1} R z_{2} /\left(2 z_{T}\right)$,

where $z_{T}=z_{0}+z_{1}+z_{2}, \quad R=p_{1} / p_{2}$, and finally $z_{\lambda}=p_{1}^{2} / 2 \lambda$ is half the Talbot distance of the first grating.

In order for a pseudoimage with significant modulation exists, the argument of the sinc function must be zero [11]. The condition $\eta\left(n-n^{\prime}\right)+\tau\left(m-m^{\prime}\right)=0$ can be written, in terms of the parameters of the problem, as
$z_{2}=\frac{1}{R Q-1} z_{1}$

where $Q=-M / N=-\left(m-m^{\prime}\right) /\left(n-n^{\prime}\right)$ is a rational number. According to this equation, given any pair of gratings with a ratio of periods $R$, a rational number $Q=-M / N$ defines a straight line in the plane $\left(z_{1}, z_{2}\right)$ with a slope $(R Q-1)^{-1}$.We call this straight line pseudoimage of $\operatorname{order}(M, N)$.

For sinusoidal gratings the only Fourier coefficients different from zero are $a_{-1}=b_{-1}=$ $a_{1}=b_{1}=1 / 4, a_{0}=b_{0}=1 / 2$ and, therefore, the number of terms in Eq. (1) is 81 . We have reordered these terms according to $N$ and $M$, since $\operatorname{sinc}[\eta N+\tau M]$ governs the location of pseudoimages, and different values for $N, M$ produces different pseudoimages. As both $N$ and $M$ run from -2 to 2 , there are 25 pairs $(N, M)$. However, the pairs $(N, M)$ and $(-N,-M)$ correspond to the same pseudoimage, as their slopes are coincident. As a consequence, the number of pseudoimages is reduced to 13: $(0,0),(0,1),(0,2),(1,-2),(1,-1)$, $(1,0),(1,1),(1,2),(2,-2),(2,-1),(2,0),(2,1)$, $(2,2)$. The irradiance at the imaging plane $P$ is then

$I(x)=\frac{1}{128} \sum T_{|N|, M}$,

where

$$
\begin{aligned}
T_{0,0}= & 18 \\
T_{0,1}= & 8 \cos \left[\Gamma R\left(z_{0}+z_{1}\right)\right] \operatorname{sinc}\left[W R z_{2}\right] \\
& \times \cos \left[2 \Omega R^{2} z_{2}\left(z_{0}+z_{1}\right)\right]\left[2+\cos \left(2 \Omega R z_{0} z_{2}\right)\right], \\
T_{0,2}= & 2 \cos \left[2 \Gamma R\left(z_{0}+z_{1}\right)\right] \operatorname{sinc}\left(2 W R z_{2}\right) \\
& \times\left[2+\cos \left(4 \Omega R z_{0} z_{2}\right)\right] \\
T_{1,-2}= & 4 \cos \left\{\Gamma\left[(1-2 R) z_{0}-2 R z_{1}\right]\right\} \\
& \times \operatorname{sinc}\left\{W\left[z_{1}+(1-2 R) z_{2}\right]\right\} \\
& \times \cos \left\{2 \Omega\left[z_{1}+(1-R) z_{2}\right] z_{0}\right\} \\
T_{1,-1}= & 8 \cos \left\{\Gamma\left[(R-1) z_{0}+R z_{1}\right]\right\} \\
& \times \operatorname{sinc}\left\{W\left[z_{1}+(1-R) z_{2}\right]\right\} \\
& \times \cos \left\{\Omega R\left[(1-2 R) z_{0}-2 R z_{1}\right] z_{2}\right\} \\
& \times \cos \left\{\Omega z_{0}\left[(R-2) z_{2}-2 z_{1}\right]\right\}, \\
T_{1,0}= & 8 \cos \left(\Gamma z_{0}\right) \operatorname{sinc}\left[W\left(z_{1}+z_{2}\right)\right] \\
& \times \cos \left[2 \Omega z_{0}\left(z_{1}+z_{2}\right)\right]\left[2+\cos \left(2 \Omega R z_{0} z_{2}\right)\right],
\end{aligned}
$$




$$
\begin{aligned}
T_{1,1}= & 8 \cos \left\{\Gamma\left[(R+1) z_{0}+R z_{1}\right]\right\} \\
& \times \operatorname{sinc}\left\{W\left[z_{1}+(1+R) z_{2}\right]\right\} \\
& \times \cos \left\{\Omega R\left[(1+2 R) z_{0}+2 R z_{1}\right] z_{2}\right\} \\
& \times \cos \left\{\Omega z_{0}\left[(R+2) z_{2}+2 z_{1}\right]\right\}, \\
T_{1,2}= & 4 \cos \left\{\Gamma\left[(1+2 R) z_{0}+2 R z_{1}\right]\right\} \\
& \times \operatorname{sinc}\left\{W\left[z_{1}+(1+2 R) z_{2}\right]\right\} \\
& \times \cos \left\{2 \Omega\left[z_{1}+(1+R) z_{2}\right] z_{0}\right\}, \\
T_{2,-2}= & \cos \left\{2 \Gamma\left[(R-1) z_{0}+R z_{1}\right]\right\} \\
& \times \operatorname{sinc}\left\{2 W\left[z_{1}+(1-R) z_{2}\right]\right\}, \\
T_{2,-1}= & 4 \cos \left\{\Gamma\left[(R-2) z_{0}+R z_{1}\right]\right\} \\
& \times \operatorname{sinc}\left\{W\left[2 z_{1}+(2-R) z_{2}\right]\right\} \\
& \times \cos \left\{2 \Omega R\left[(R-1) z_{0}+R z_{1}\right] z_{2}\right\}, \\
T_{2,0}= & 2 \cos \left(\Gamma z_{0}\right) \\
& \times \operatorname{sinc}\left[2 W\left(z_{1}+z_{2}\right)\right]\left[2+\cos \left(4 \Omega R z_{0} z_{2}\right)\right], \\
T_{2,1}= & 4 \cos \left\{\Gamma\left[(2+R) z_{0}+R z_{1}\right]\right\} \\
& \times \operatorname{sinc}\left\{W\left[2 z_{1}+(2+R) z_{2}\right]\right\} \\
& \times \cos \left\{2 \Omega R\left[(1+R) z_{0}+R z_{1}\right] z_{2}\right\}, \\
T_{2,2}= & \cos \left\{2 \Gamma\left[(R+1) z_{0}+R z_{1}\right]\right\} \\
& \times \operatorname{sinc}\left\{2 W\left[z_{1}+(1+R) z_{2}\right]\right\}, \\
&
\end{aligned}
$$

with $\Gamma=x q_{1} / z_{T}, W=S q_{1} / 2 z_{T}$, and $\Omega=\pi / 4 z_{T} z_{\lambda}$.

Each of these 13 terms (except $T_{0,0}$ which is constant) present four multiplicative factors: (1) A constant value, related to the Fourier coefficients of both gratings, which determines the maximum amplitude of the pseudoimage. (2) A sinc factor which determines the pseudoimage location $\left(z_{1}, z_{2}\right)$ and the relation between its width and the source size. (3) A cosine factor with an argument depending linearly on $x$, and which determines its spatial period and (4) one or several cosine factors, which determine the modulation of the pseudoimage and depends on the periods of the gratings, distances involved and wavelength.

\section{Analysis of pseudoimages}

As stated in Eq. (2), the location of the pseudoimages depends on the $Q$ factor. Two pseudoimages with the same $Q$ value overlap. Overlapping of pseudoimages with different but similar $Q$ values will depend on the extension of the region in the plane $\left(z_{1}, z_{2}\right)$ where the sinc function reaches significant values, which in turn depends on the ratio $S q_{1} / z_{T}$.

The distances $z_{1}$ and $z_{2}$ must take positive values, so for a pseudoimage to be real the slope $(R Q-1)^{-1}$ must be positive. The meaning of a negative value of the slope is that the pseudoimage is virtual, and henceforth its contribution to the irradiance at any plane with $z_{2}>0$ is negligible. From Eq. (3), we can see that only the pseudoimages $T_{1,-1}, T_{2,-2}, T_{1,-2}$, and $T_{2,-1}$ have positive slopes (and, as we have just explained, the first two overlap). If we assume that $S q_{1} \gg 1$, the pseudoimages $T_{1,-1}, T_{1,-2}$ and $T_{2,-1}$ do not overlap, and the total intensity $I_{Q}(x)$ around them is approximately the sum of the constant $T_{0,0}$ term and the intensity provided by such pseudoimages, that is

$$
\begin{aligned}
& I_{1 / 2}(x) \approx \frac{1}{128}\left(T_{0,0}+T_{2,-1}\right), \\
& I_{1}(x) \approx \frac{1}{128}\left(T_{0,0}+T_{1,-1}+T_{2,-2}\right), \\
& I_{2}(x) \approx \frac{1}{128}\left(T_{0,0}+T_{1,-2}\right) .
\end{aligned}
$$

This approximation introduces an error of about $8 \%$ in the computation of irradiance, with respect to the exact result given by Eq. (3). A comparison between the approximate and the exact values of irradiance along the pseudoimage $I_{2}$ is shown in Fig. 4, for $p_{1}=p_{2}=20 \mu \mathrm{m}, S=300$ $\mu \mathrm{m}, \lambda=600 \mathrm{~nm}$ and $z_{0}=0$. In Fig. 2 , the contrast obtained from Eq. (3) is shown for $p_{1}=20 \mu \mathrm{m}$, $R=3, S=300 \mu \mathrm{m}$ and $\lambda=600 \mathrm{~nm}$. The contrast is defined as

$C=\frac{I_{\max }-I_{\min }}{I_{\max }+I_{\min }}$,

where $I_{\max }$ and $I_{\min }$ are respectively the maximum and minimum irradiance on the observation plane. For this configuration, the three pseudoimages $I_{1 / 2}, I_{1}$ and $I_{2}$ appear, with slopes $1 / 2,2$, and 5 respectively. Modulation and contrast of pseudoimages $I_{1 / 2}$ and $I_{1}$ strongly oscillates along the straight lines $z_{2}=z_{1} / 2$, and $z_{2}=2 z_{1}$, but pseudoimage $I_{2}(x)$ is stable. In order to determine the conditions for which the pseudoimages are stable, we calculate the irradiance distribution along the 


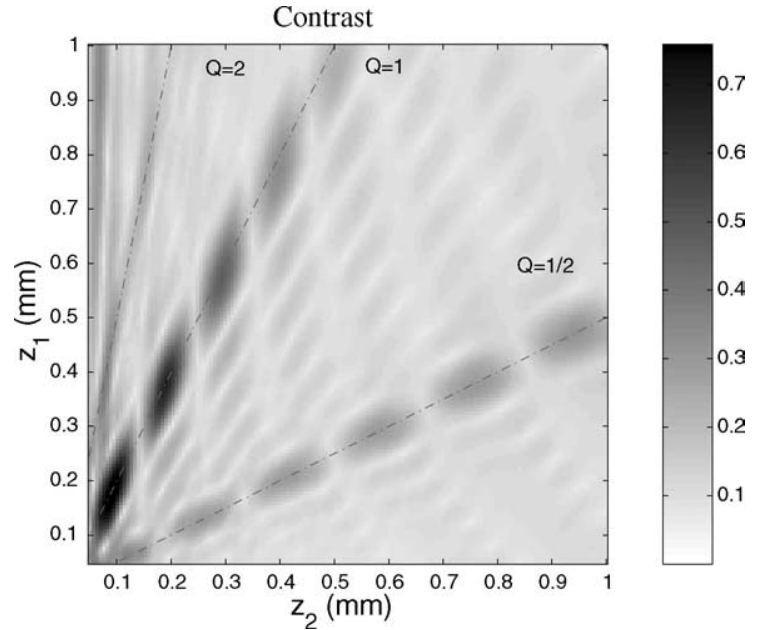

Fig. 2. Contrast obtained using Eq. (3) for two sinusoidal gratings of period $p_{1}=20 \mu \mathrm{m}, p_{2}=20 / 3 \mu \mathrm{m}(R=3)$. The light source $(S=300 \mu \mathrm{m}, \lambda=600 \mathrm{~nm})$ is placed at $z_{0}=1 \mathrm{~mm}$. Three pseudoimages $(Q=2,1$, and $1 / 2)$ appear. Pseudoimages $Q=1$ and $Q=1 / 2$ present strong fluctuations in contrast, but pseudoimage $Q=2$ is not so fluctuating.

straight line of each pseudoimage. By making the substitution $z_{1}=(R Q-1) z_{2}$ in Eq. (4) we obtain

$$
\begin{aligned}
I_{1 / 2}^{p}\left(x, z_{2}\right) \approx & \frac{9}{64}+\frac{1}{32} \cos \left[x q_{1}(R-2)\right] \\
& \times \cos \left[\frac{\pi R}{2 z_{\lambda}} \frac{(R-1)+R(R-2) z_{2} /\left(2 z_{0}\right)}{z_{2}^{-1}+(R / 2) z_{0}^{-1}}\right], \\
I_{1}^{p}\left(x, z_{2}\right) \approx & \frac{9}{64}+\frac{1}{128} \cos \left[2 x q_{1}(R-1)\right] \\
& +\frac{1}{16} \cos \left[x q_{1}(R-1)\right] \\
& \times \cos \left[\frac{\pi R}{4 z_{\lambda}} \frac{(1-2 R)-2 R(R-1) z_{2} / z_{0}}{z_{2}^{-1}+z_{0}^{-1} R}\right] \\
& \times \cos \left[\frac{\pi R}{4\left(z_{2}^{-1}+z_{0}^{-1} R\right) z_{\lambda}}\right], \\
I_{2}^{p}\left(x, z_{2}\right) \approx & \frac{1}{64}\left\{9+2 \cos \left[x q_{1}(2 R-1)\right]\right. \\
& \left.\times \cos \left(\frac{\pi R}{2 z_{\lambda}} \frac{1}{z_{2}^{-1}+2 R z_{0}^{-1}}\right)\right\} .
\end{aligned}
$$

It is worth to note that $T_{2,-2}$ is stable, but its modulation is small and it is masked by the term $T_{1,-1}$, which is 8 times larger.

By determining the minima of the last cosine factor of $I_{1 / 2}^{p}(x)$ and $I_{1}^{p}(x)$, we can see that the irradiance fluctuation cannot be eliminated. In the case of $I_{2}^{p}(x)$, the minima of the pseudoimage modulation are located at

$z_{2} \|_{Q=2}^{\min }=\frac{z_{0} z_{\lambda} / R}{z_{0} /(1+2 L)-2 z_{\lambda}}, \quad$ where

$$
L=\ldots,-1,0,1, \ldots
$$

When $z_{0}>2 z_{\lambda}$ there are positive values of $z_{2} \|_{Q=2}^{\min }$, and irradiance fluctuations still appear. In the limiting case $z_{0} \rightarrow \infty$, the minima are equidistantly distributed at $z_{2} \|_{Q=2}^{\min }=(1+2 L) z_{\lambda} / R$. On the other hand, when $z_{0} \leqslant 2 z_{\lambda}$ there are no positive solutions to $z_{2} \|_{Q=2}^{\min }$ and, as a consequence, $I_{2}^{p}\left(x, z_{2}\right)$ depends very slowly on $z_{2}$ and a stable pseudoimage is obtained. To our knowledge, this effect has not been reported before.

An example of this behaviour is shown in Fig. 3, for which we have chosen $p_{1}=p_{2}=20 \mu \mathrm{m}$, $S=300 \mu \mathrm{m}, \lambda=600 \mathrm{~nm}$ and $z_{0}=0$. The approximate and exact irradiance profiles along the stable pseudoimage $I_{2}$ shown in Fig. 3 are presented in Fig. 4. The error made when we use the isolated terms $T_{0,0}$ and $T_{1,-2}$ to compute the contrast of $I(x)$ along the straight line $z_{1}=z_{2}$ is about $8 \%$. The exact profile is not completely flat, as the neglected pseudoimages contribute and oscillate in this

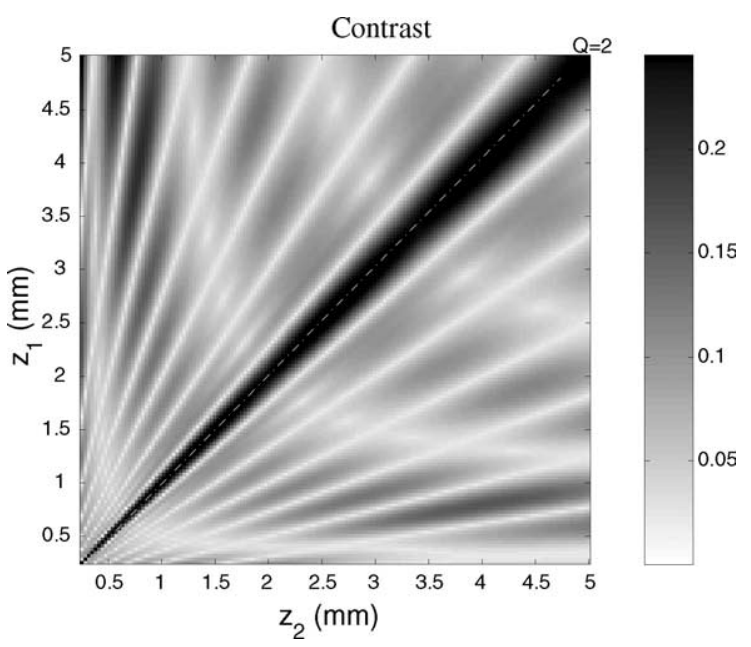

Fig. 3. Contrast obtained using Eq. (3) and two sinusoidal gratings of period $p_{1}=p_{2}=20 \mu \mathrm{m}(R=1)$. The light source $(S=300 \mu \mathrm{m}, \lambda=600 \mathrm{~nm})$ is placed at $z_{0}=0 \mu \mathrm{m}$. Only the pseudoimage $Q=2$ is present, and it is continuous, that is, there is not a decay in contrast. 


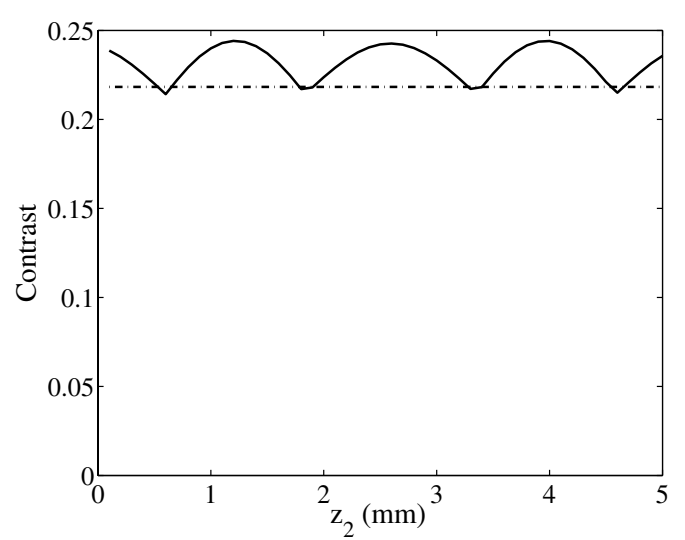

Fig. 4. Comparison between the contrast obtained by Eq. (3) (---) and Eq. (4) (-. - .) for configuration of Fig. 3, and at the exact pseudoimage location $\left(z_{1}=z_{2}\right)$. The contrast obtained by the two equations is quite similar.

region. In any case, this small riple is far more easy to tolerate than the full oscillation of pseudoimages $I_{1}$ and $I_{1 / 2}$.

In order to take advantage of the reported stability of pseudoimages generated with sinusoidal gratings, a synchronized variation of $z_{1}$ and $z_{2}$ is necessary. Double transmission grating systems are not indicated to take advance of this property. Instead, $G 2$ can be made a reflection grating, so that the observation plane may coincide with the first grating. With this configuration, it is easy to guarantee that $z_{1}=z_{2}$, whatever the particular value of $z_{1}$ could be.

\section{Conclusions}

We have analyzed generalized grating imaging for the case of two sinusoidal gratings illuminated with an extended monochromatic light source. A simple analytical expression is obtained for the intensity of pseudoimages, and it is shown that for certain conditions of source location one of the pseudoimages is stable along its profile in the plane $\left(z_{1}, z_{2}\right)$. The modulation and contrast of these stable pseudoimages is bound to smaller values than those achieved with Ronchi gratings in oscillating pseudoimages. This reduction in contrast can be easily overcome with adequate electronics. On the other hand, the strong contrast fluctuation that is produced by variation in the gap between gratings makes the ordinary pseudoimages very sensitive to optomechanical tolerances. The pseudoimage stabilization has potential interest in the design and manufacturing of scientific and measuring equipment using generalized pseudoimaging (Talbot spectrometers and interferometers, moire based measuring devices, displacement measuring sensors, etc.), as tolerances in grating positioning can be enormously increased.

\section{Acknowledgements}

This project was supported by the Ministerio de Ciencia y Tecnología of Spain projects DPI20011238 and DPI2001-1369.

\section{References}

[1] F. Talbot, Philos. Mag. 9 (1836) 401.

[2] K. Patorski, in: E. Wolf (Ed.), Progress in Optics, 27, North Holland, Amsterdam, 1989, p. 3.

[3] E. Keren, O. Kafri, J. Opt. Soc. Am. A 2 (1985) 111.

[4] F.O. Weinberg, N.B. Wood, J. Sci. Instr. 36 (1959) 227.

[5] E. Lau, Ann. Phys. (Leipzig) 6 (1985) 417.

[6] J. Jahns, A.W. Lohmann, Opt. Commun. 28 (1979) 263.

[7] K. Patorski, Opt. Acta 30 (1983) 745.

[8] G.J. Swanson, E.N. Leith, J. Opt. Soc. Am. A 2 (1985) 789.

[9] J. Tu, L. Zhan, J. Opt. Soc. Am. A 9 (1992) 983.

[10] A. Olszak, L. Wronkowski, Opt. Eng. 36 (1997) 2149.

[11] D. Crespo, J. Alonso, E. Bernabeu, J. Opt. Soc. Am. A 17 (2000) 1231.

[12] D. Crespo, J. Alonso, E. Bernabeu, Appl. Opt. 41 (2002) 1223.

[13] N. Guérineau, B. Harchaoui, J. Primot, Opt. Commun. $180(2000) 199$.

[14] N. Guerineau, B. Harchaoui, J. Primot, K. Heggarty, Opt. Lett. 26 (2001) 411.

[15] S. Teng, L. Liu, J. Zu, Z. Luan, De'an Liu, J. Opt. Soc. Am. A 20 (2003) 1747. 